\title{
A Case of Septic Arthritis of the Shoulder Joint That Developed after Suprascapular Nerve Block
}

\author{
Taihei Go ${ }^{1}$, Toshiyuki Tsutsui ${ }^{*}$, Yasuaki Iida ${ }^{3}$, Katsunori Fukutake ${ }^{1}$, Ryoichi Fukano ${ }^{3}$, \\ Kosei Ishigaki ${ }^{4}$, Masayuki Sekiguchi ${ }^{1}$, Hiroshi Takahashi ${ }^{1}$ \\ ${ }^{1}$ Department of Orthopedics, Toho University, Tokyo, Japan \\ ${ }^{2}$ Department of Orthopedics, Sagamihara Chuo Hospital, Kanagawa, Japan \\ ${ }^{3}$ Department of Orthopedics, Omori Red Cross Hospital, Tokyo, Japan \\ ${ }^{4}$ Department of Orthopedics, Japan Community Health Care Organization Tokyo Kamata Medical Center, Tokyo, Japan \\ Email: taiheigo@gmail.com, *tsutsui-ortho@tokujukai.or.jp, yasuaki.iida@med.toho-u.ac.jp, \\ fukutakekatsunori04077@gmail.com,ryouichi.fukano@med.toho-u.ac.jp, k.ishigaki0304@gmail.com, \\ masa1961@med.toho-u.ac.jp, drkan@med.toho-u.ac.jp
}

How to cite this paper: Go, T., Tsutsui, T., Iida, Y., Fukutake, K., Fukano, R., Ishigaki, K., Sekiguchi, M. and Takahashi, H. (2020) A Case of Septic Arthritis of the Shoulder Joint That Developed after Suprascapular Nerve Block. Open Journal of Orthopedics, 10, 25-32.

https://doi.org/10.4236/ojo.2020.102005

Received: November 30, 2019

Accepted: January 19, 2020

Published: January 22, 2020

Copyright $\odot 2020$ by author(s) and Scientific Research Publishing Inc. This work is licensed under the Creative Commons Attribution International License (CC BY 4.0).

http://creativecommons.org/licenses/by/4.0/

\begin{abstract}
Septic arthritis of the shoulder is uncommon in the immunocompetent patient with no previous risk factors for joint infection. We treated an immunocompetent patient who developed septic arthritis of the shoulder after suprascapular nerve block for pain due to rotator cuff tear. An 80-year-old man with no underlying disease visited a nearby orthopedics clinic with complaint of left shoulder joint pain. Left suprascapular nerve block was performed, but the pain gradually aggravated. On the day after the block, he had a fever of $39^{\circ} \mathrm{C}$ and came to our department. On examination, enlargement and tenderness were present at the injection site. Cellulitis at the site was suspected. He was admitted and administration of a cephem antibiotic was started. Pain subsequently decreased. Magnetic resonance imaging (MRI) performed 4 days after hospitalization showed massive effusion close to the injection site. The effusion spread into the joint cavity through the tear site of the supraspinatus. Septic arthritis of the shoulder was strongly suspected, open irrigation and debridement were performed 11 days after hospitalization. After surgery, pain immediately improved. In our case the extra-articular infection caused by suprascapular nerve block considered to spread into the shoulder joint cavity through the site of rotator cuff tear, although there have been no reports of such cases. This case suggests the possibility that patients with rotator cuff tear may easily develop septic arthritis because extra-articular infection may spread into the joint cavity through the site of tear.
\end{abstract}




\section{Keywords}

Septic Arthritis of the Shoulder Joint, Suprascapular Nerve Block, Rotator Cuff Tear

\section{Introduction}

Septic arthritis of the shoulder is relatively rare (approximately $3 \%$ of all joint infection) [1] but is a serious and difficult-to-cure disease in which joint function may be damaged without early treatment [2] [3] [4]. The disease normally develops in elderly people with underlying disease after antecedent infection or a minor invasion such as arthrocentesis [1] [2] [5]. Leslie et al. reported that in patients with septic arthritis of the shoulder, hematogenous causes were responsible for $56 \%$ of cases, intra-articular procedures for $11 \%$, and steroid injection for $33 \%$ [2]. In contrast, there have been no reports of cases that developed after suprascapular nerve block. Here, we report a case of septic arthritis of the shoulder that suspected to develop after suprascapular nerve block in an elderly man with no underlying disease. We also include a discussion of the literature.

\section{Case Presentation}

The patient was an 80-year-old man with a major complaint of left shoulder joint pain. He had no specific medical history. He had become aware of left shoulder joint pain several years ago, but the pain resolved spontaneously at that time. In August 2018, left shoulder joint pain redeveloped without any trauma. Since the pain did not resolve in several days, he visited a nearby orthopedics clinic, where left suprascapular nerve block was performed for the pain. We confirmed later that $5 \mathrm{ml}$ of $1 \%$ xylocaine was injected using a 21-gauge needle after sterilization with povidone-iodine. Subsequently, the left shoulder joint pain gradually aggravated. On the day after the block, he had a fever of $39^{\circ} \mathrm{C}$ and came to our department.

At the first visit, body temperature was $38.7^{\circ} \mathrm{C}$, blood pressure was $138 / 97$ $\mathrm{mmHg}$, pulse rate was $80 / \mathrm{min}$, and the patient was lucid. Enlargement and tenderness were present at the injection site for left suprascapular nerve block, but enlargement of the left shoulder joint was mild (Figure 1). The pain was severe, as shown by a visual analogue scale (VAS) of 10/10 points, and both autokinetic and passive movement of the left shoulder joint was difficult. The Japanese Orthopedic Association (JOA) Score, the University of California at Los Angeles (UCLA) score, and the Constant Shoulder Score were 20/100, 4/35, and 8/100 points, respectively.

The white blood cell (WBC) count was $14,140 / \mu$, the C-reactive protein (CRP) level was $32.3 \mathrm{mg} / \mathrm{dl}$, and sedimentation was $104 / 60 \mathrm{~min}$, indicating a strong inflammatory reaction. There were no other clear abnormalities in bio- 
chemical and hematological tests. Plain X-ray images obtained at admission showed superior translation of the bone head, but no abnormal findings such as bone lysis or bone head subluxation (Figure 2).

Enlargement and tenderness were mainly present at the injection site for left suprascapular nerve block, cellulitis at the site was suspected. He was admitted and administration of a cephem antibiotic was started at a dose of $3 \mathrm{~g} /$ day that pain subsequently decreased (VAS 7/10 points). Administration of an antibiotic was started without submitting any bacterial cultures. In magnetic resonance imaging (MRI) of the left shoulder joint performed 4 days after hospitalization, T1- and T2-weighted images showed low and high intensity areas around the supraspinatus and infraspinatus close to the injection site for suprascapular nerve block, respectively, suggesting significant accumulation of liquid. The liquid spread from the tear site of the supraspinatus into the joint cavity and then to the synovial bursa located anterior to the shoulder joint. Septic arthritis of the shoulder was strongly suspected based on the clinical course and MRI findings of the patient (Figure 3).

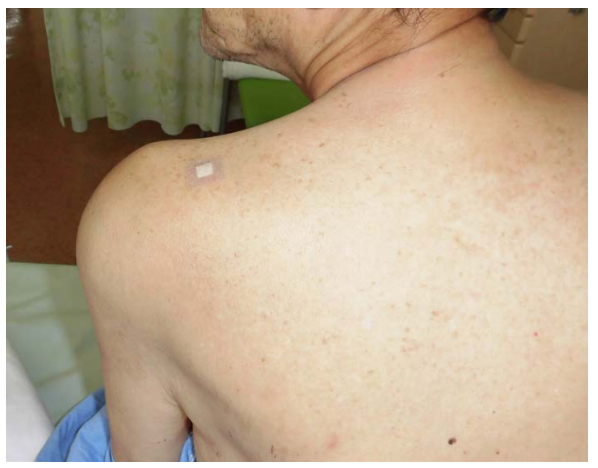

Figure 1. Enlargement and tenderness were confirmed at the injection site for left suprascapular nerve block. A plaster was applied at the site.

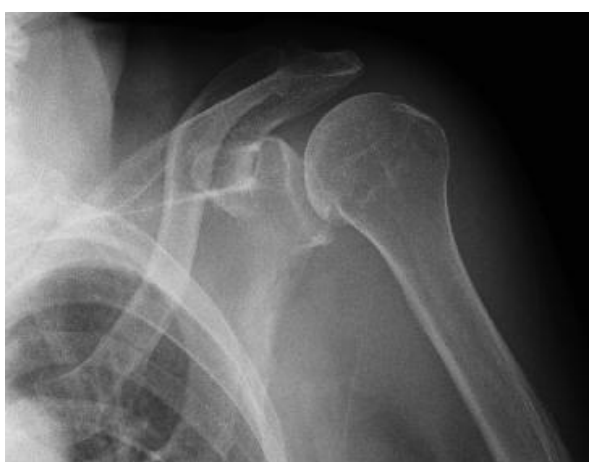

Figure 2. Plain X-ray frontal image at admission. Superior translation of the bone head was observed, but there were no abnormal findings such as bone lysis and subluxation of the bone head. 


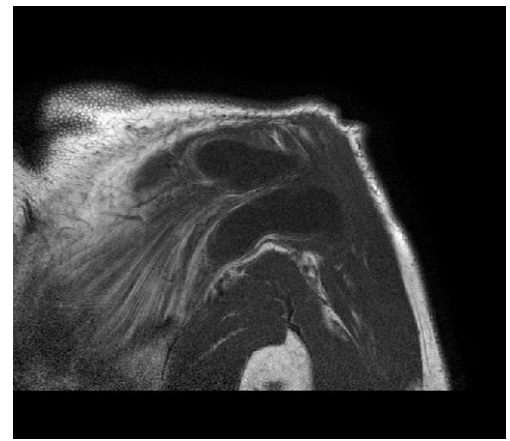

(a)

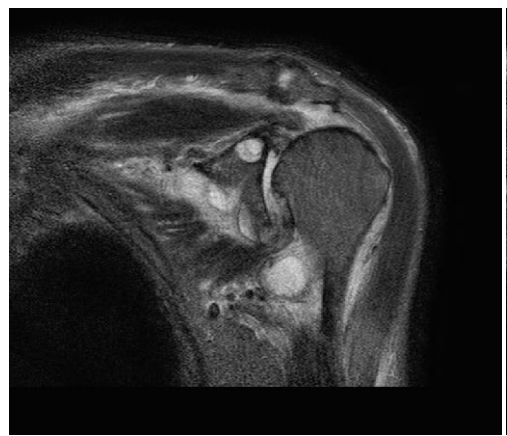

(c)

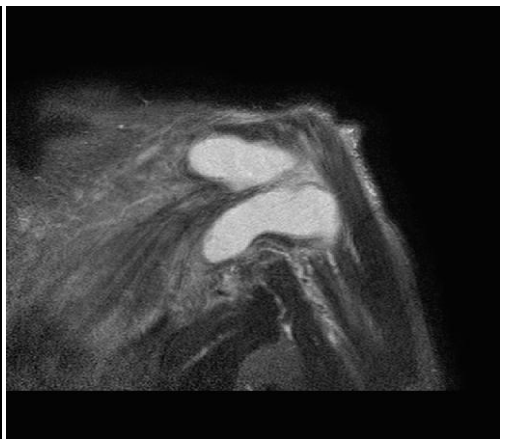

(b)

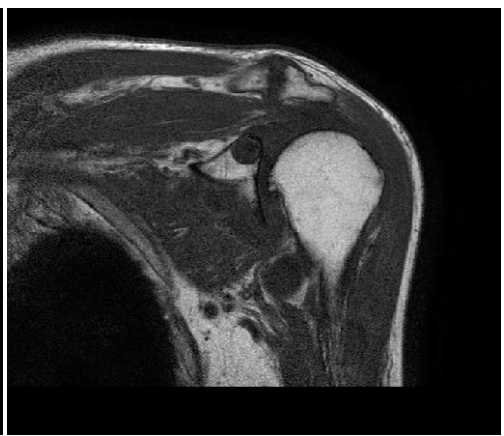

(d)

Figure 3. MR coronal cut image of the left shoulder joint taken on day 4 of hospitalization. Low intensity in T1-weighted images (a) and high intensity in T2-weighted and STIR images (b) suggested significant accumulation of liquid in the area around the supraspinatus and infraspinatus, close to the injection site for suprascapular nerve block. The liquid shown as low intensity on T1-weighted images (c) and as high intensity on STIR images (d) spread from the tear site of the supraspinatus into the joint cavity and then to the synovial bursa located anterior to the shoulder joint.

In blood sampling 9 days after hospitalization, WBC had decreased to $5690 / \mu \mathrm{l}$, but CRP was $12.7 \mathrm{mg} / \mathrm{dl}$, suggesting insufficient improvement. Open irrigation and debridement were performed 11 days after hospitalization. Based on the clinical and imaging findings, the extra-articular injection site for suprascapular nerve block was thought to be the main infected lesion, and thus surgery was performed with open approach. After incision of the supraspinatus and infraspinatus, cloudy serous pus and necrotic tissues were confirmed (Figure 4), which were removed as much as possible and the site was thoroughly cleansed with saline. The amount of pus was small, and the infection had been improved to a certain extent by the antibiotic. After partial incision of the joint capsule, a small amount of pus leaked from the joint cavity (Figure 4). A Teflon I.V. Catheter was injected at the site and irrigation was performed using 2 liters of saline. All samples of tissues from the supraspinatus and infraspinatus, serous liquid, and joint fluid collected during surgery were negative in bacterial culture.

After surgery, pain immediately improved (VAS $1 / 10$ points) and CRP normalized to $0.3 \mathrm{mg} / \mathrm{dl}$ at 4 weeks after surgery with continuous administration of the cephem antibiotic (Figure 5). At 3.5 months after onset, pain was almost resolved and the shoulder joint could be flexed for $130^{\circ}$, abducted for $110^{\circ}$, externally ro- 
tated for $40^{\circ}$, and internally rotated to Th12. The JOA, UCLA and Constant Shoulder scores improved from 20/100, 4/35 and 8/100 points at admission to $83 / 100,26 / 35$ and $57 / 100$ points at 3.5 months after onset, respectively. At the last examination at 1.5 years after onset, no redevelopment of infection was found.

\section{Discussion}

Septic arthritis develops mainly in the knees and hip joints (61\% - 79\%), which carry the load of the lower extremities, but is rare in shoulder joints (approx. $3 \%)$ [1]. However, the rate of septic arthritis of the shoulder has increased to $10 \%-15 \%$ of all types of arthritis in recent years due to increased use of arthroscopy and advanced aging [5]. In many cases, septic arthritis of the shoulder develops in patients with chronic underlying diseases, such as diabetes, blood disorder, renal failure, malignant tumor, malnutrition, and rheumatoid arthritis; after administration of immunosuppressive drugs, such as long-term administration of steroids; or after a minor invasion, such as arthrocentesis and antecedent infection. Some cases with infection after surgery for rotator cuff injury have been described in relatively young patients with no underlying disease, and hematogenous infection in infants has also been reported [2] [4] [6]. However, to the best of our knowledge, there has been no previous report of septic arthritis that developed after suprascapular nerve block.

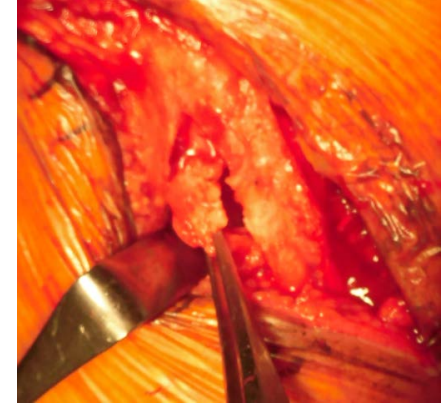

(a)

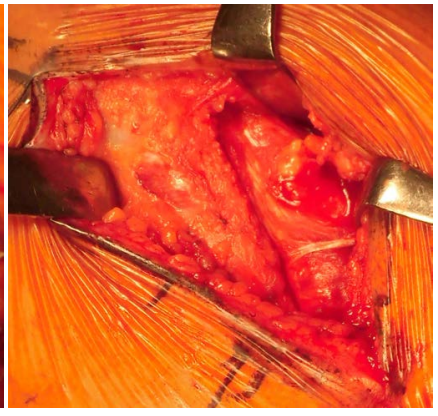

(b)

Figure 4. After incision of the supraspinatus (a) and infraspinatus (b), cloudy serous pus and necrotic tissues were observed. The photo of the left shoulder was taken from the dorsal side. The right and left sides show the head and caudal sides, respectively.

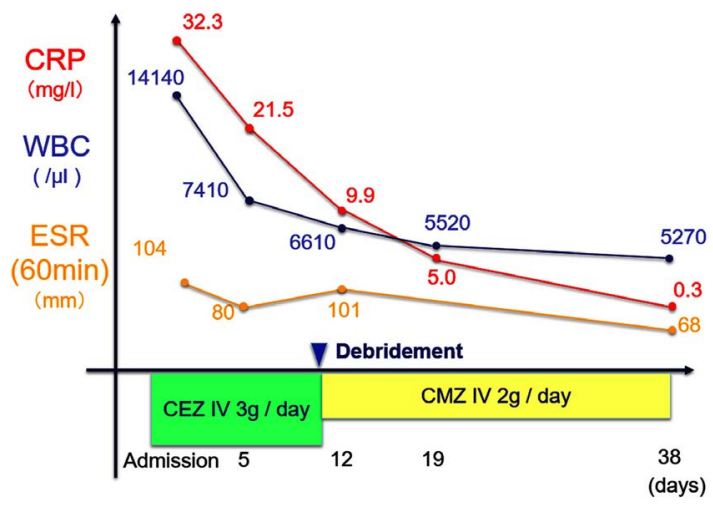

Figure 5. Clinical course after admission. 
Our patient had pain at a site close to the injection site for suprascapular nerve block as the major symptom at the first visit. In addition, cellulitis was suspected because the patient had not received an injection in the joint cavity of the shoulder, and thus we did not perform joint puncture due to a risk of secondary infection caused by this procedure. Leslie et al. reported that symptoms such as reddening, enlargement and a sensation of burning were not observed in shoulder joints of some patients with septic arthritis of the shoulder, but aggravation of pain upon both passive and autokinetic movement was found in all cases [2]. Our case also had no apparent reddening, enlargement or sensations of burning, but pain of the shoulder joint was severe upon both passive and autokinetic movement and a strong inflammatory reaction was present. Thus, arthrocentesis should have been performed, because the possibility of expansion of infection to the shoulder joint thought to be high. Administration of an antibiotic was started before the bacterial cultures were obtained, and all the results were negative. Since pain and inflammation improved immediately after initiation of administration of a cephem antibiotic, septic arthritis was strongly suspected based on the clinical course and MRI finding of the patient.

Regarding the infection route, since infection was not confirmed at the other sites, the possibility of blood infection can be excluded. After suprascapular nerve block, the symptom developed at a site close to the injection site, and MRI also showed most accumulation of liquid at a site close to the nerve block injection site. This liquid communicated with the joint cavity at the site of supraspinatus tear. Based on these findings, we conclude that the suprascapular nerve block injection site became infected first, and then the infection expanded to the joint cavity and caused septic arthritis of the shoulder. The subacromial bursa and subdeltoid bursa communicate with the joint cavity of the shoulder in patients with rotator cuff tear, and septic arthritis of the shoulder can spread from the site of rotator cuff tear to the extra-articular subacromial bursa, causing septic subacromial bursitis [7] [8]. However, the extra-articular infection spread into the shoulder joint cavity through the site of rotator cuff tear in our case, suggesting an inverse infection route. The patient became aware of shoulder joint pain several years ago, and the pain resolved spontaneously. Plain X-ray images obtained at the first visit to our department confirmed an elevated humeral head. These findings suggest that the rotator cuff tear was initially asymptomatic, but subsequently became symptomatic, which led to the need for suprascapular nerve block.

Most reports suggest that septic arthritis of the shoulder can be treated by concomitant surgery and administration of antibiotics. Use of arthroscopic debridement, which is less invasive and enables early-stage rehabilitation, has tended to increase, but arthroscopic and open surgery show similarly favorable results. However, there are no established treatment guidelines [6]. As patients with extra-articular abscess formation or bone destruction in a wide area have a high risk of recurrence after arthroscopic surgery, open surgery should be con- 
sidered as a treatment option in such cases [9] [10]. Since the extra-articular injection site for suprascapular nerve block was the major infection site in our case, we selected treatment with open debridement and irrigation. The rate of reoperation after arthroscopic irrigation performed within 2 weeks after development is lower than that with irrigation at a later time, and the functional prognosis is better [9] [11]. In our case, we performed open surgery 11 days after hospitalization, and the prognosis was favorable.

An epidemiological study based on medical checkups in Japan found rotator cuff tear in approx. $25 \%, 45 \%$ and $50 \%$ of persons in their 60 s, 70 s and 80 s, respectively [12]. The incidence of rotator cuff tear as a complication of septic arthritis of the shoulder is high: Klinger et al. [9], Jeon et al. [11], and Yamagata et al. [10] reported rotator cuff tear in 14 of 23 patients (approx. 61\%, average age: 63.7 years), 11 of 19 patients (approx. 58\%, average age: 59 years), and 8 of 12 patients (approx. 67\%, average age: 74.2 years) with septic arthritis of the shoulder, respectively. All of these were retrospective studies. Although a direct causal relationship between rotator cuff tear and septic arthritis of the shoulder is unclear, Jeon et al. performed joint injection and steroid injection in 15 and 11 of 19 patients, respectively. Since the frequency of joint injection is high in patients with rotator cuff tear, the risk of iatrogenic infection may also be high.

Our case received suprascapular nerve block at a nearby hospital, and we confirmed later that $5 \mathrm{ml}$ of $1 \%$ xylocaine was injected using a 21-gauge needle after sterilization with povidone-iodine. The rates of septic arthritis after joint injection and arthroscopic surgery are 4 and 14 per 10,000 cases, respectively [13]. No previous reports have suggested septic arthritis after suprascapular nerve block, which indicates the rarity of this condition. Since our case had no underlying disease, it is possible that the technique for suprascapular nerve block in the nearby hospital might have been inappropriate, or the injection agent might have been contaminated.

\section{Conclusion}

We experienced the case of septic arthritis of the shoulder developed with the patient who had rotator cuff tear after left suprascapular nerve block. This case suggests the possibility that patients with rotator cuff tear may easily develop septic arthritis because extra-articular infection may spread into the joint cavity through the site of tear.

\section{Consent}

The patient gave informed consent to submit this case study for publication.

\section{Conflicts of Interest}

The authors declare that there is no conflict of interest regarding the publication of this paper. 


\section{References}

[1] Lossos, I.S., et al. (1998) Septic Arthritis of the Glenohumeral Joint. A Report of 11 Cases and Review of the Literature. Medicine, 77, 177-187. https://doi.org/10.1097/00005792-199805000-00003

[2] Leslie, B.M., et al. (1989) Septic Arthritis of the Shoulder in Adults. Journal of Bone and Joint Surgery, 71-A, 1516-1522. https://doi.org/10.2106/00004623-198971100-00010

[3] Bremell, T., et al. (1992) Histopathological and Serological Progression of Experimental Staphylococcus aureus Arthritis. Infection and Immunity, 60, 2976-2985. https://doi.org/10.1128/IAI.60.7.2976-2985.1992

[4] Wick, M., et al. (2003) Arthrodesis of the Shoulder after Septic Arthritis. Long-Term Results. Journal of Bone and Joint Surgery, 85-B, 666-670. https://doi.org/10.1302/0301-620X.85B5.13915

[5] Abdou, M.A., et al. (2019) Shoulder Joint Infections with Negative Culture Results: Clinical Characteristics and Treatment Outcomes. BioMed Research International, 2019, Article ID: 3756939. https://doi.org/10.1155/2019/3756939

[6] Kenji, Y., et al. (2013) Treatment of 17 Cases of Septic Arthritis of the Shoulder. Bone Joint Nerve, 3, 739-745.

[7] Michimasa, U.I., et al. (2004) The Clinical Results of a Septic Arthritis of the Shoulder. The Shoulder Joint (JPN), 28, 583-587.

[8] Matsui, K., et al. (1992) A Case of Acute Septic Arthritis of the Shoulder Caused by Arthrography. Clinical Orthopaedic Surgery $(J P N), 27,167-169$.

[9] Joen, I.H., et al. (2006) Arthroscopic Management of Septic Arthritis of the Shoulder Joint. Journal of Bone and Joint Surgery, 88, 1802-1806. https://doi.org/10.2106/JBJS.E.00917

[10] Yamagata, H., et al. (2015) Therapeutic Experience of Septic Arthritis of the Shoulder Joint. Orthopedics \& Traumatology, 64, 506-509. https://doi.org/10.5035/nishiseisai.64.506

[11] Klinger, H.M., et al. (2010) Septic Arthritis of the Shoulder Joint. An Analysis of Management and Outcome. Acta Orthopaedica Belgica, 76, 598-603.

[12] Yamamoto, A., et al. (2010) Prevalence and Risk Factors of a Rotator Cuff Tear in the General Population. Journal of Shoulder and Elbow Surgery, 19, 116-120. https://doi.org/10.1016/j.jse.2009.04.006

[13] Mathews, C.J., et al. (2010) Bacterial Septic Arthritis in Adults. Lancet, 375, 846-855. https://doi.org/10.1016/S0140-6736(09)61595-6 